\title{
Experience-related remapping of temporal encoding by striatal ensembles.
}

R. Austin. Bruce ${ }^{1,2}$, Matthew A. Weber ${ }^{2}$, Rachael A. Volkman², Mayu Oya ${ }^{2}$, Eric B. Emmons ${ }^{3}$, Youngcho $\mathrm{Kim}^{2}$, and Nandakumar S. Narayanan ${ }^{2 *}$

\author{
Affiliations: \\ ${ }^{1}$ Interdisciplinary Graduate Program in Neuroscience, University of Iowa, Iowa City, IA, 52242 \\ ${ }^{2}$ Department of Neurology, University of Iowa, Iowa City, IA 52242 \\ ${ }^{3}$ Department of Biology, Wartburg College, Waverly, IA, 50677
}

Abstract: 204

Figures: 5

*Corresponding Author

Nandakumar Narayanan

nandakumar-narayanan@uiowa.edu

169 Newton Road

Pappajohn Biomedical Discovery Building-1336

University of Iowa, Iowa City, 52242

319-353-5698 


\begin{abstract}
Temporal control of action is key for a broad range of behaviors and is disrupted in human diseases such as Parkinson's disease and schizophrenia. A brain structure that is critical for temporal control is the dorsal striatum. Experience and learning can influence dorsal striatal neuronal activity, but it is unknown how these neurons change with experience in contexts which require precise temporal control of movement. We investigated this question by recording from medium-spiny neurons (MSNs) in the dorsal striatum of mice as they gained experience controlling their actions in time. We leveraged an interval timing task optimized for mice which required them to "switch" response ports after enough time had passed without receiving a reward. We report three main results. First, we found that time-related ramping activity and response-related activity increased with more experience. Second, temporal decoding by MSN ensembles improved with experience and was predominantly driven by time-related ramping activity. Finally, we found that some MSNs had differential modulation on error trials. These findings enhance our understanding of dorsal striatal temporal processing by demonstrating how MSN ensembles can evolve with experience. Our results can be linked to temporal habituation and illuminate striatal flexibility during interval timing, which may be relevant for human disease.
\end{abstract}




\section{Introduction}

Precisely guiding movements in time is critical for human behaviors, such as cooking,

26 driving, and crossing the street (Buhusi \& Meck, 2005). Temporal control of action is disrupted

27 in diseases such as Parkinson's disease (PD) and schizophrenia (Ward et al., 2011; Parker et al.,

28 2013; Singh et al., 2021). A brain structure that is affected by both diseases and is key for

29 controlling movements in time is the dorsal striatum (Matell \& Meck, 2004; Meck, 2006). Thus,

30 understanding how the dorsal striatum contributes to the timing of movement is important for

31 human disease.

32 Dorsal striatal circuits can be explored in detail in rodent models. In rodents, disrupting

33 striatal dopamine profoundly impairs the temporal control of action (Meck, 2006; Wang et al.,

34 2018). Striatal neurons encode time on the scale of several seconds (Matell et al., 2003; Mello et

35 al., 2015; Bakhurin et al., 2017; Emmons et al., 2017; Wang et al., 2018). For example, striatal

36 neurons can encode temporal information by time-dependent ramping, in which firing rate

37 changes monotonically over a temporal interval (Emmons et al., 2017), although other temporal

38 encoding schemes may also be involved (Paton and Buonomano, 2018; Zhou et. al 2020).

39 Task-evoked activity in striatal ensembles remaps with behavioral experience. Human

40 brain imaging studies have found that the activity of dorsal striatal networks can be increased

41 with sustained experience in operant tasks (Tricomi et al., 2009). Moreover, dorsal striatal

42 activity adapts with learning of specific contexts and tasks, while disrupting dorsal striatal

43 activity can impair previously learned associations (Pasupathy \& Miller, 2005; Yin et al., 2005;

44 Yin \& Knowlton, 2006). The activity of striatal neurons changes markedly with sustained

45 experience in the same procedural task even when behavior is relatively constant (Barnes et al., 
46 2005). This literature predicts that temporal encoding in the dorsal striatum should evolve with

47 experience.

48 Here, we tested the hypothesis that striatal ramping changes as animals gain experience

49 over 10 days of performing an interval timing task. We recorded from dorsal striatal ensembles

50 of putative medium-spiny neurons (MSNs) in mice trained to perform an interval timing task

51 which required them to "switch" from one response port to another after an interval (Balci,

52 Ludvig, et al., 2008; Balci, Papachristos, et al., 2008; Tosun et al., 2016). We report three main

53 results: 1) time-related ramping patterns of activity among MSNs increased with experience; 2)

54 temporal decoding of MSNs improved with experience; and 3) trials with erroneous responses

55 had distinct patterns of MSN activity. Our results provide insight into how temporal encoding in

56 striatal MSNs evolves with experience.

57

58

59 
61 Mice

62 All procedures were approved by the Institutional Animal Care and Use Committee (IACUC) at

63 the University of Iowa, and all methods were performed in accordance with the relevant

64 guidelines and regulations (Protocol \#0062039). Mice were motivated by 85\% food restriction.

Interval-timing switch task

67 Mice were trained to perform a switch interval timing task. Operant chambers (MedAssociates,

68 St. Albans, VT) enclosed in sound-attenuating cabinets were equipped with two nosepokes on

69 one wall, a food delivery port (reward hopper) located between them, a back nosepoke opposite

70 the food delivery port, and a speaker that produced an $8 \mathrm{kHz}$ tone at $72 \mathrm{~dB}$. Mice were rewarded

71 with 20-mg sucrose pellets (Bio-Serv, Flemington, NJ). First, animals were shaped to nosepoke

72 in response to rewards using fixed-ratio task trials. These trials were initated when a mouse

73 responded at the back nosepoke, followed by delivery of a reward after the mouse responded at

74 an illuminated nosepoke on either side of the reward hopper. Trials were counterbalanced so that

75 animals learned to respond at nosepokes on either the left or right side of the reward hopper.

76 Subsequently, mice were advanced to the switch interval timing task. This task consisted of short

77 trials ( $50 \%$ of trials) and long trials (50\% of trials). During short trials mice were rewarded for a

78 response at the designated short nosepoke (either the left or right nosepoke). During long trials, a

79 response at the short nosepoke was unreinforced; instead, a response at the nosepoke on the

80 contralateral side of the reward hopper (long nosepoke) was rewarded after 18 seconds. The

81 nosespokes associated with short and long trials were counterbalanced to the left or right port

82 across mice and were randomly intermixed, and the start of each trial was indicated by the same 
83 tone. Optimally, mice started by responding at the short nosepoke and switching to the long

84 nosepoke sometime around or after six seconds (Fig 1A). The mouse's decision to switch from

85 the short nosepoke to the long nosepoke is a time-based decision, guided by the temporal control

86 of action. Only long switch trials were analyzed.

87 Following implantation of striatal recording arrays (see below), animals were initially

88 trained in the switch interval timing task for a period of 18 days and then acclimated to recording

89 procedures. Recordings from the first day with $>5$ completed trials were considered "early

90 training." Mice were then trained to perform the switch interval timing task for an additional 10

91 days prior to recording in the "experienced" session. Mice received intraperitoneal drug

92 injections on days 5 and 7.

93

94 Surgical and histological procedures

95 C57BL/6 mice (Jackson Labs, Bar Harbor, ME) were anesthetized using IP injections of

96 ketamine $(100 \mathrm{mg} / \mathrm{kg})$ and xylazine $(10 \mathrm{mg} / \mathrm{kg})$, and a surgical level of anesthesia was

97 maintained using ketamine and xylazine supplements (10 mg/kg and $1 \mathrm{mg} / \mathrm{kg}$, respectively).

98 Craniotomies were drilled above the dorsal striatum (Fig 1B), and holes were drilled for skull

99 screws that were connected to electrode recording arrays (MicroProbes, Gaithersburg, MD) via a

100 separate ground wire. Microelectrode arrays were composed of $4 \times 450-\mu \mathrm{m}$ stainless steel wires

101 (250 $\mu \mathrm{m}$ between wires and rows). These arrays were positioned in the dorsal striatum

102 (coordinates from bregma: AP +0.9, ML $\pm 1.4, \mathrm{DV}-2.7 @ 0^{\circ}$ in the posterior or lateral plane; Fig

103 1B) while recording neuronal activity to verify that implantation was in the correct brain area.

104 The craniotomy was sealed with cyanoacrylate (“SloZap,” Pacer Technologies, Rancho

105 Cucamonga, CA), and the reaction was accelerated by "ZipKicker" (Pacer Technologies) and 
methyl methacrylate (AM Systems, Port Angeles, WA). Mice recovered for one week before

107 being acclimated to behavioral and recording procedures. Only the left striatum was implanted in

108 all mice.

109 Following these procedures, posterolateral recording array corners (electrode \#16) were

110 marked by electrolytic lesions induced by applying a current of 50 microamps over 10 seconds

111 through the array. After four days, mice were anesthetized using ketamine (100 $\mathrm{mg} / \mathrm{kg} \mathrm{IP})$ and

112 xylazine (10 mg/kg IP) and transcardially perfused with 4\% formalin. Brains were post-fixed in a

113 solution of $4 \%$ formalin and $30 \%$ sucrose, before being horizontally sectioned on a freezing

114 microtome. Brain slices were mounted on Superfrost Plus microscope slides (Thermo Fisher

115 Scientific, Waltham, MA) and stained for cell bodies using either cresyl violet, or nuclear fast

116 red solution / Prussian Blue for electrode localization. Histological reconstruction was completed

117 using postmortem analysis of electrode placement by slide-scanning light microscopy (Fig 1B;

118 Olympus, Center Valley, PA).

Neurophysiological recordings and neuronal analyses

121 Neuronal ensemble recordings were made using a multi-electrode recording system

122 (Plexon, Dallas, TX). In each mouse, one electrode without single units was reserved for local 123 referencing, yielding 15 electrodes per animal. After the experiments, Offline Sorter software

124 (Plexon) was used to analyze the signals and to remove artifacts. Spike activity was analyzed for 125 all cells that fired at rates above $0.1 \mathrm{~Hz}$. Principal Component Analysis (PCA) and waveform 126 shape were used for spike sorting. Single units were defined as those 1) having a consistent 127 waveform shape, 2) being a separable cluster in PCA space, and 3) having a consistent refractory 128 period of at least 2 milliseconds in interspike interval histograms. Putative MSNs were further 
129 separated from striatal interneurons based on hierarchical clustering of the waveform peak-to-

130 trough ratio and the half-peak width (fitgmdist and cluster.m; Fig 1C) (Berke, 2011). All

131 neuronal analyses focused on putative MSNs. Note that the same electrodes are recorded from

132 across sessions.

134 Statistics

135 All data and statistical approaches were reviewed by the Biostatistics, Epidemiology, and 136 Research Design Core (BERD) at the Institute for Clinical and Translational Sciences (ICTS) at

137 the University of Iowa. All code and data are available at http://narayanan.lab.uiowa.edu. For

138 behavioral data, we used linear mixed-effects models where the outcome variable was response

139 time on long trials and the predictor variable was session type ("early training" vs.

140 "experienced"); animals were included as a random effect. We used the median to measure

141 central tendency and the interquartile range to measure variance across mice. Wilcoxon

142 nonparametric signed-rank or rank-sum tests were used to compare behavior between early

143 training and experienced sessions. Wilxocon signed-rank tests were also used to compare

144 decoding analyses between early training and experienced sessions and different neuronal

145 ensembles. To quantify effect size, we used Cohen's d. To present evidence related to the null

146 hypothesis, we calculated the Bayes Factor (Morey \& Rouder, 2011).

$147 \quad$ Analyses of neuronal activity and basic firing properties were carried out using

148 NeuroExplorer (Nex Technologies, Littleton, MA) and custom routines for MATLAB (Parker et

149 al., 2014; Emmons et al., 2017, 2019). Neuronal modulations were quantified using generalized

150 linear models (GLMs) at the individual neuron level. For each neuron, we constructed a model in

151 which the response variable was the firing rate binned at 0.2 seconds and the predictor variable 
152 was either time in the interval or motor response. All models were run at a trial-by-trial level to

153 avoid effects of trial-averaging (Latimer et al., 2015). For each neuron, a p-value was obtained

154 for main effects of time in the interval (i.e., "time-related ramping") or response. Across the

155 ensemble, p-values were corrected via Benjamini-Hochberg false-discovery-rate (FDR), with

156 values $<0.05$ considered significant for each neuron. To identify neurons with different activity

157 at short vs. long or right vs. left nosepokes, we compared firing rates in the 0.2 seconds around

158 nosepoke response via a Wilcoxon rank-sum test for each neuron. We compared GLM-defined

159 neuronal modulations between early training and experienced sessions via a $\mathrm{X}^{2}$ test, assuming

160 neurons were independent as in our past work (Narayanan \& Laubach, 2006; Parker et al., 2014,

161 2015; Emmons et al., 2017; Kim et al., 2017). For comparisons of ramping-related or response-

162 related modulations between early training and experienced sessions, we used logistic regression

163 and included a random intercept for each animal to control for mouse-specific effects. For error-

164 related modulations, a similar approach was taken, but the GLM was constructed where the

165 reponse variable was firing rate, and the predictor variable was whether the trial was correct or

166 had erroneous nosepokes; only neurons from animals with $>5$ trials of each type were analyzed.

167 A p-value was obtained for main effects of errors for each neuron and corrected via FDR across

168 the ensemble.

169 We used a naïve Bayesian classifier to examine neuronal ensemble decoding, as we have

170 in our past work (Emmons et al., 2017; Kim et al., 2017). We calculated kernel density estimates

171 (bandwidth: 0.5) of trial-by-trial MSN firing rates, binned at 1 second, from all neurons with at

172 least eight trials. To prevent edge effects that might bias classifier performance, we included data

173 from six seconds prior to trial start and six seconds after interval end. We used leave-one-out

174 cross-validation to predict an objective time from firing rate within a trial. We evaluated 
175 classifier performance by computing the $\mathrm{R}^{2}$ of objective time vs. predicted time, only for bins

176 during the interval. With perfect classification, the $\mathrm{R}^{2}$ would approach 1 . Classifier performance

177 was compared to ensembles with time-shuffled firing rates via a Wilcoxon signed-rank test.

178

179 


\section{Results}

Our goal was to study how striatal temporal encoding evolves with experience. We tested this idea by implanting multielectrode recording arrays targeting the dorsal striatum and training

183 six animals in the switch interval timing task for $\sim 10$ days (Fig 1A). For animals in early

184 training, the average short nosepoke response time was $9.3 \pm 2.8$ seconds (median \pm interquartile

185 range) and $13.7 \pm 3.3$ seconds at the long nosepoke. For experienced animals, the average

186 response times were $7.5 \pm 1.4$ seconds at the short nosepoke and $14.8 \pm 1.2$ seconds at the long 187 nosepoke. Mice did not reliably perform more trials in experienced sessions compared to early 188 training (31 \pm 26 trials in early training vs. $39 \pm 21$ trials in experienced sessions, Wilcoxon signed189 rank $\mathrm{p}=0.13$, Bayes factor $=0.74$ ). A linear mixed-effects model revealed that there was a highly190 significant main effect of short vs. long nosepoke $\left(\mathrm{F}_{(1,2788)}=27.7, \mathrm{p}<0.05\right)$, a main effect of

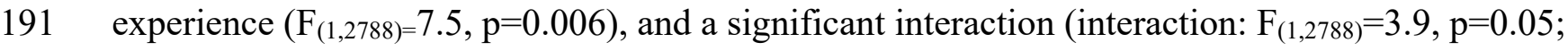

192 model fit $\mathrm{R}^{2}=0.20$; Fig $\left.2 \mathrm{~A}-\mathrm{E}\right)$. Although there was no consistent change in switch times $(9.3 \pm 3.6$ 193 seconds vs. $9.3 \pm 1.5$ seconds; Wilcoxon signed-rank $\mathrm{p}=0.8$; Bayes factor=2.5; Fig $2 \mathrm{~F}$ ) or the

194 coefficient in variation $(36.3 \pm 17.9 \%$ vs. $34.5 \pm 20.8 \%$; Fig $2 \mathrm{G})$, mice performed better with 195 experience, with a higher percentage of trials terminated by a response at the correct response 196 port (naïve: $59 \pm 22 \%$ vs. experienced $80 \pm 23 \%$; Wilcoxon signed-rank p=0.03; Cohen's d=1.3; 197 Fig 2H).

198 We were interested in medium spiny neuron (MSN) activity during the switch interval199 timing task. We recorded from 77 MSNs in early training and 79 MSNs in the same animals that 200 had become experienced after extensive training. To quantify experience effects at a trial-by-trial 201 level, we turned to GLMs in which the outcome variable was firing rate and the predictor 202 variable was either time in the interval or motor response. Neurons with an FDR-corrected 
203 significant main effect of time were considered to exhibit time-related ramping activity (Fig 3A),

204 and neurons with FDR-corrected significant main effects of response were considered response-

205 related (Fig 3B). Additionally, many neurons displayed both time-dependent ramping and

206 response-related changes in their activity (Fig 3C). We found that the total number of time-

207 related ramping neurons increased with experience $\left(29 \%\right.$ vs. $44 \%, X_{(1,156)}^{2}=4.2, p=0.04 ; p=0.01$

208 via mixed-effect logistic regression; Fig 3C-D), as did the total number of response-related

209 neurons $\left(28 \%\right.$ vs. $46 \% ; \mathrm{X}^{2}{ }_{(1,156)}=5.6, \mathrm{p}=0.02 ; \mathrm{p}=0.01$ via mixed-effect logistic regression; Fig

210 3C-D). Additionally, the number of neurons that showed both time-related ramping and

211 response-related modulation increased with experience $\left(10 \%\right.$ vs. $25 \% ; X_{(1,156)}^{2}=6.0, p=0.01 ;$ Fig

212 3C-D). Of note, there were response-related neurons that were modulated by short vs. long

213 responses, but these did not significantly change with experience (14 of 21 response-related

214 neurons in early training and 16 of 36 neurons in experienced sessions had short vs. long

215 activity; $\left.\mathrm{X}_{(1,57)}^{2}=2.6, \mathrm{p}=0.11\right)$. We found that $9 \%$ of neurons in early training sessions were

216 modulated by the switch response, when mice transitioned from short to long nosepokes, and

$21710 \%$ were modulated in experienced sessions $\left(\mathrm{X}_{(1,156)}^{2}=0.2, \mathrm{p}=0.66\right)$. These data suggest that

218 ramping increased with experience, both on its own and where it interacted with response-related

219 activity.

220 To examine the computational consequences of this shift, we turned to decoding analyses

221 where we used naïve Bayesian classification to predict time from striatal ensembles (Emmons et

222 al., 2017; Kim et al., 2017). Consistent with the change in time-related ramping with experience,

223 we found that experienced striatal ensembles predicted time with greater accuracy than in early

224 training $\left(\mathrm{R}^{2}\right.$ : early training: $0.29 \pm 0.41$ vs. experienced: $0.62 \pm 0.60$; Wilcoxon signed-rank $\mathrm{p}=0.02$;

225 Cohen's d=0.8; Fig 4A-C). Of note, both ensembles were reliably more predictive of time than 
shuffled data (early training shuffled $\mathrm{R}^{2}$ : 0.01 \pm 0.04 ; Wilcoxon signed-rank vs. early training $\mathrm{p}=0.0002$; experienced shuffled $\mathrm{R}^{2}: 0.11 \pm 0.18$; Wilcoxon signed-rank vs. early training $\mathrm{p}=0.0005)$. In experienced sessions we found that MSNs with both time-related ramping and response-related activity were similarly predictive of time (ramping $\mathrm{R}^{2}: 0.38 \pm 0.38$; ramping $\&$ response $\mathrm{R}^{2}$ : $0.47 \pm 0.35$; Wilcoxon signed-rank $\mathrm{p}=0.28$; Bayes factor $=3.0$; data from experienced sessions only; Fig. 4C). Critically, this was stronger than MSNs with response-related activity alone $(0.10 \pm 0.31$; Wilcoxon signed-rank $\mathrm{p}=0.001$ vs. ramping; Cohen's $\mathrm{d}=1.2 ; \mathrm{p}=0.007$ vs. ramping\&response:; Cohen's d=0.9; Fig. 4D). Furthermore, striatal ensembles without time-

234 related ramping did not decode time well $(0.14 \pm 0.18$; Wilcoxon signed-rank $\mathrm{p}=0.0003$ vs.

235 ramping; Cohen's d=1.4; p=0.002 vs. time-related ramping\&response; Cohen's d=1.1; Fig. 4D).

236 These data make three key points: 1) ensembles with time-related ramping contributed strongly

237 to temporal decoding, whether or not they had response-related activity, 2) ensembles without

238 time-related ramping had poor temporal decoding, and 3) response-related neuronal activity,

239 despite increasing with experience, did not decode time. These data are consistent with previous

240 findings from our group in that neurons with time-related ramping strongly convey temporal

241 information (Emmons et. al., 2017) and that temporal decoding improves in striatal ensembles

242 with experience (Emmons et. al., 2020).

Finally, we examined neuronal activity on error trials in which animals did not

244 successfully switch from short to long nosepokes (Fig 5). We only examined neurons that had

245 five or more error trials; we recorded 61 neurons in early training and 37 neurons in experienced

246 sessions among our six mice. In early training, 51\% of these neurons were error-modulated,

247 which was not consistently different from experienced sessions $\left(35 \%, \mathrm{X}_{(1,98)}^{2}=2.8, \mathrm{p}=0.10\right.$; Fig

248 5B). These data indicate that error trials could involve distinct patterns of striatal activity but that 
249 these patterns did not consistently incfease with experience. Together, these results together

250 describe how ramping modulations, response modulations, and temporal decoding increase with

251 experience while error-related activity does not.

252

253 


\section{Discussion}

255 We explored how striatal temporal encoding remapped with experience. First, we found 256 that patterns of MSN activity evolved after 10 days of interval timing. Second, we found that

257 dorsal striatal ensembles improved temporal decoding with experience. This was driven largely

258 by MSNs with time-related ramping activity, as MSNs without this activity had poor temporal

259 decoding. Finally, we found that dorsal striatal neurons could have distinct patterns of error-

260 related activity. Taken together, these data provide evidence that MSN temporal encoding can

261 evolve with experience.

262 These data are broadly consistent with our previous work (Emmons et al., 2016, 2017,

$2632019,2020)$. In one of these studies with well-trained animals (Emmons et al., 2020), we found

264 that striatal time-related ramping was stable as highly-trained rats learned a new, shorter fixed

265 interval over three days. By contrast, in this study we compared striatal temporal encoding just

266 after mice aquired a switch interval timing task and again after $\sim 10$ days of experience. We note

267 that striatal decoding improved in both studies, providing convergent evidence that temporal

268 encoding by striatal ensembles is enhanced with experience.

269 Movement confounds studies of the temporal control of action (Namboodiri \& Hussain

270 Shuler, 2014; Paton \& Buonomano, 2018). We have previously controlled for motor confounds

271 via regression (Parker et al., 2014; Kim et al., 2017; Emmons et al., 2019), and we note that

272 time-related ramping is still observed prior to the first response (Emmons et al., 2017). In switch

273 interval timing, response rates at the short interval do not monotonically increase with time;

274 indeed response rates at the long interval increase over the interval but plateau in experienced

275 animals, and switch interval times are mostly distributed after six seconds (Fig 2D); Balci,

276 Papachristos, et al., 2008). Supporting this idea are our observations that response-related 
277 activity alone did not decode time (temporal decoding was driven by time-related ramping

278 activity), and that neurons multiplexing time and movement could change with experience.

279 Finally, time-related ramping can reflect averaging across a session (Latimer et al., 2015). For

280 this reason, our definition of time-related ramping was derived from trial-by-trial GLMs that

281 quantify trial-by-trial main effects of firing rate over the interval (Emmons et al., 2017), although

282 additional analytic tools may help further resolve these dynamics (Narayanan, 2016; Zylberberg

283 \& Shadlen, 2016).

There are many alternative coding schemes that robustly represent temporal information,

285 including those based on oscillatory activity, sequences, and network states (Paton \&

286 Buonomano, 2018; Zhou et al., 2020). While those features may be latent within our dorsal

287 striatal ensembles, we have reported consistent evidence that time-related ramping contributes to

288 temporal decoding (Narayanan \& Laubach, 2009; Parker et al., 2014; Narayanan, 2016; Emmons

289 et al., 2017; Kim et al., 2017). We have also shown that striatal ramping is correlated with

290 prefrontal ramping and requires prefrontal top-down control (Emmons et al., 2017, 2019). While

291 other coding schemes may be relevant, we found that striatal ensembles without ramping activity

292 decode time poorly. Taken together, these data suggest that time-related ramping contributes to

293 temporal processing in tasks that require temporal control of action.

294 We report that striatal neurons exhibit different activity profiles on error trials in which

295 animals failed to switch from the short to the long nosepoke. This analysis was not possible in

296 our previous work with the fixed-interval timing task or in many prior studies of interval timing

297 in which all trials with responses after a target interval were rewarded (Matell et al., 2003; Parker

298 et al., 2014, 2015; Bakhurin et al., 2017; Emmons et al., 2017, 2020). In addition, despite

299 increases in time-related ramping, response-related activity and fewer errors with experience, 
300 error-related activity did not change with experience. This finding suggests that error-related

301 firing is not strictly related to time-related ramping or responding; future studies may further

302 elucidate these error-related patterns, particulary in contexts when mice make enough errors to

303 facilitate robust analyses.

304 Finally, these data illuminate the neuronal basis of temporal habits. Indeed, the dorsal

305 striatum is critical for habits in which stimulus-response associations persist in overtrained

306 animals, despite changes in reward contingencies (Yin et al., 2005; Yin \& Knowlton, 2006;

307 Balleine et al., 2007). Accordingly, past work has found that the striatum profoundly remaps

308 with overtraining (Barnes et al., 2005). We did not manipulate rewards in the present study, as

309 we found relatively sparse reward representations in our striatal ensembles. However, we found

310 profound remapping in striatal ensemble encoding of movements and time, which may represent

311 the neural basis of temporal habits. This idea is supported by our behavioral data suggesting that

312 switch time and variation do not consistently change with experience. Future studies will explore

313 how reward devaluation might influence interval timing and other stimulus-response associations

314 separated by time.

315 Dorsal striatal neurons encode many aspects of behavior (Kimchi et al., 2009; Kimchi \&

316 Laubach, 2009). We report that time-related ramping increases with experience during interval

317 timing. This knowledge is germane to human diseases that affect the striatum such as

318 Parkinson's disease (PD). Indeed, PD patients can have specific challenges in learning (Frank et

319 al., 2004; Nieuwboer et al., 2009) and in performing functions for which they have extensive

320 experience (Uc et al., 2006, 2007). Understanding the striatal basis of temporal encoding might

321 lead to new treatments for human diseases such as Parkinson's disease and schizophrenia that

322 degrade learning, cognition, and previously-formed habits. 
Our work has several limitations. The first is that we cannot dissociate MSN subtypes

324 that express D1 vs. D2 dopamine receptors, and we are uncertain how the powerful

325 neuromodulator dopamine affects striatal networks (Cui et al., 2013). Second, we cannot fully

326 dissociate ramping activity from movements, which would require alternative task designs

327 (Church \& Deluty, 1977), although one critical advantage of our task design is that it readily

328 translates to humans (Ward et al., 2011; Kim et al., 2017; Parker et al., 2017), particularly with

329 Parkinson's disease (Singh et al., 2021). Third, with our approach, we were unable to identify

330 oscillatory or state-based coding schemes in the striatum that change with experience, although

331 our analytical approach may not be suited to identify these network properties.

332 Despite these limitations, the present study provides evidence that temporal encoding in

333 the dorsal striatum changes with experience. Specifically, we find that time-related ramping can

334 increase, resulting in improved temporal decoding. Our results illustrate how striatal ensembles

335 remap with temporal experience. 
339 Acknowledgements:

340 This work was funded by NIH MH116043 to NSN. This study was supported in part by the

341 University of Iowa Institute for Clinical and Translational Science, which is granted with

342 Clinical and Translational Science Award funds from the National Institutes of Health

343 (UL1TR002537).

346 Author contributions:

347 RAB and NSN designed the experiments. RAB collected the data, with assistance from RAV,

$348 \mathrm{MO}$, and MAW. RAB and YK wrote the code. EE and YK checked the analysis and code. RAB,

349 MAW, and NSN wrote the paper. 


\section{References}

Bakhurin, K.I., Goudar, V., Shobe, J.L., Claar, L.D., Buonomano, D.V., \& Masmanidis, S.C. (2017) Differential Encoding of Time by Prefrontal and Striatal Network Dynamics. $J$. Neurosci., 37, 854-870. Balci, F., Ludvig, E.A., Gibson, J.M., Allen, B.D., Frank, K.M., Kapustinski, B.J., Fedolak, T.E., \& Brunner, D. (2008) Pharmacological manipulations of interval timing using the peak procedure in male C3H mice. Psychopharmacology (Berl), 201, 67-80. Balci, F., Papachristos, E.B., Gallistel, C.R., Brunner, D., Gibson, J., \& Shumyatsky, G.P. (2008) Interval timing in genetically modified mice: a simple paradigm. Genes Brain Behav, 7, 373384. and decision-making. The Journal of Neuroscience, 27, 8161-8165. Barnes, T.D., Kubota, Y., Hu, D., Jin, D.Z., \& Graybiel, A.M. (2005) Activity of striatal neurons reflects dynamic encoding and recoding of procedural memories. Nature, 437, 1158-1161. Berke, J.D. (2011) Functional properties of striatal fast-spiking interneurons. Front Syst Neurosci, 5, 45.

Buhusi, C.V. \& Meck, W.H. (2005) What makes us tick? Functional and neural mechanisms of interval timing. Nat. Rev. Neurosci, 6, 755-765.

Church, R.M. \& Deluty, M.Z. (1977) Bisection of temporal intervals. J Exp Psychol Anim Behav Process, 3, 216-228.

Cui, G., Jun, S.B., Jin, X., Pham, M.D., Vogel, S.S., Lovinger, D.M., \& Costa, R.M. (2013)

Concurrent activation of striatal direct and indirect pathways during action initiation. Nature, 494, 238-242.

Emmons, E.B., Corte, B.J.D., Kim, Y., Parker, K.L., Matell, M.S., \& Narayanan, N.S. (2017) Rodent Medial Frontal Control of Temporal Processing in the Dorsomedial Striatum. $J$. Neurosci., 37, 8718-8733. Emmons, E.B., Kennedy, M., Kim, Y., \& Narayanan, N.S. (2019) Corticostriatal stimulation compensates for medial frontal inactivation during interval timing. Scientific Reports, 9, 1-9. Frank, M.J., Seeberger, L.C., \& O'Reilly, R.C. (2004) By Carrot or by Stick: Cognitive Reinforcement Learning in Parkinsonism. Science, 306, 1940-1943. Kim, Y.-C., Han, S.-W., Alberico, S.L., Ruggiero, R.N., De Corte, B., Chen, K.-H., \& Narayanan, N.S. (2017) Optogenetic Stimulation of Frontal D1 Neurons Compensates for Impaired Temporal Control of Action in Dopamine-Depleted Mice. Curr Biol, 27, 39-47. Kimchi, E.Y. \& Laubach, M. (2009) The dorsomedial striatum reflects response bias during learning. J. Neurosci., 29, 14891-14902. Kimchi, E.Y., Torregrossa, M.M., Taylor, J.R., \& Laubach, M. (2009) Neuronal correlates of instrumental learning in the dorsal striatum. J. Neurophysiol., 102, 475-489. Latimer, K.W., Yates, J.L., Meister, M.L.R., Huk, A.C., \& Pillow, J.W. (2015) NEURONAL MODELING. Single-trial spike trains in parietal cortex reveal discrete steps during decisionmaking. Science, 349, 184-187.

392 Matell, M.S. \& Meck, W.H. (2004) Cortico-striatal circuits and interval timing: coincidence detection of oscillatory processes. Brain Res Cogn Brain Res, 21, 139-170. Matell, M.S., Meck, W.H., \& Nicolelis, M.A.L. (2003) Interval timing and the encoding of signal duration by ensembles of cortical and striatal neurons. Behavioral Neuroscience, 117, 760-773. 
mesolimbic, nigrostriatal, and mesocortical dopaminergic systems. Brain Research, 1109, 93107.

Mello, G.B.M., Soares, S., \& Paton, J.J. (2015) A Scalable Population Code for Time in the Striatum. Current Biology, 25, 1113-1122.

Namboodiri, V.M.K. \& Hussain Shuler, M.G. (2014) Report of interval timing or action? Proc. Natl. Acad. Sci. U.S.A., 111, E2239.

Narayanan, N.S. (2016) Ramping activity is a cortical mechanism of temporal control of action. Curr Opin Behav Sci, 8, 226-230.

Narayanan, N.S. \& Laubach, M. (2006) Top-down control of motor cortex ensembles by dorsomedial prefrontal cortex. Neuron, 52, 921-931.

Narayanan, N.S. \& Laubach, M. (2009) Delay activity in rodent frontal cortex during a simple reaction time task. J. Neurophysiol, 101, 2859-2871.

Nieuwboer, A., Rochester, L., Müncks, L., \& Swinnen, S.P. (2009) Motor learning in Parkinson's disease: limitations and potential for rehabilitation. Parkinsonism \& Related Disorders, Proceedings of WFN XVIII World Congress on Parkinson's Disease and Related Disorders, 15, S53-S58.

Parker, K.L., Chen, K.-H., Kingyon, J.R., Cavanagh, J.F., \& Narayanan, N.S. (2014) D1Dependent $4 \mathrm{~Hz}$ Oscillations and Ramping Activity in Rodent Medial Frontal Cortex during Interval Timing. J. Neurosci., 34, 16774-16783.

Parker, K.L., Kim, Y.C., Kelley, R.M., Nessler, A.J., Chen, K.-H., Muller-Ewald, V.A., Andreasen, N.C., \& Narayanan, N.S. (2017) Delta-frequency stimulation of cerebellar projections can compensate for schizophrenia-related medial frontal dysfunction. Mol Psychiatry, 22, 647-655.

Parker, K.L., Lamichhane, D., Caetano, M.S., \& Narayanan, N.S. (2013) Executive dysfunction in Parkinson's disease and timing deficits. Front Integr Neurosci, 7, 75.

Parker, K.L., Ruggiero, R.N., \& Narayanan, N.S. (2015) Infusion of D1 Dopamine Receptor Agonist into Medial Frontal Cortex Disrupts Neural Correlates of Interval Timing. Front Behav Neurosci, 9, 294.

Pasupathy, A. \& Miller, E.K. (2005) Different time courses of learning-related activity in the prefrontal cortex and striatum. Nature, 433, 873-876.

Paton, J.J. \& Buonomano, D.V. (2018) The Neural Basis of Timing: Distributed Mechanisms for Diverse Functions. Neuron, 98, 687-705.

Singh, A., Cole, R.C., Espinoza, A.I., Evans, A., Cao, S., Cavanagh, J.F., \& Narayanan, N.S. (2021) Timing variability and midfrontal $\sim 4 \mathrm{~Hz}$ rhythms correlate with cognition in Parkinson's disease. npj Parkinson's Disease, 7, 1-8.

Tosun, T., Gur, E., \& Balci, F. (2016) Mice plan decision strategies based on previously learned time intervals, locations, and probabilities. Proc Natl Acad Sci U S A, 113, 787-792.

Tricomi, E., Balleine, B.W., \& O’Doherty, J.P. (2009) A specific role for posterior dorsolateral striatum in human habit learning. European Journal of Neuroscience, 29, 2225-2232.

Uc, E.Y., Rizzo, M., Anderson, S.W., Sparks, J.D., Rodnitzky, R.L., \& Dawson, J.D. (2006)

Driving with distraction in Parkinson disease. Neurology, 67, 1774-1780.

Uc, E.Y., Rizzo, M., Anderson, S.W., Sparks, J.D., Rodnitzky, R.L., \& Dawson, J.D. (2007) Impaired navigation in drivers with Parkinson's disease. Brain, 130, 2433-2440.

Wang, J., Narain, D., Hosseini, E.A., \& Jazayeri, M. (2018) Flexible timing by temporal scaling of cortical responses. Nature Neuroscience, 21, 102.

Ward, R.D., Kellendonk, C., Kandel, E.R., \& Balsam, P.D. (2011) Timing as a window on 
444 cognition in schizophrenia. Neuropharmacology,.

445 Yin, H.H. \& Knowlton, B.J. (2006) The role of the basal ganglia in habit formation. Nat Rev

446 Neurosci, 7, 464-476.

447 Yin, H.H., Ostlund, S.B., Knowlton, B.J., \& Balleine, B.W. (2005) The role of the dorsomedial

448 striatum in instrumental conditioning. European Journal of Neuroscience, 22, 513-523.

449 Zhou, S., Masmanidis, S.C., \& Buonomano, D.V. (2020) Neural Sequences as an Optimal

450 Dynamical Regime for the Readout of Time. Neuron, 108, 651-658.e5.

451 Zylberberg, A. \& Shadlen, M.N. (2016) Cause for pause before leaping to conclusions about

452 stepping. bioRxiv, 085886. 

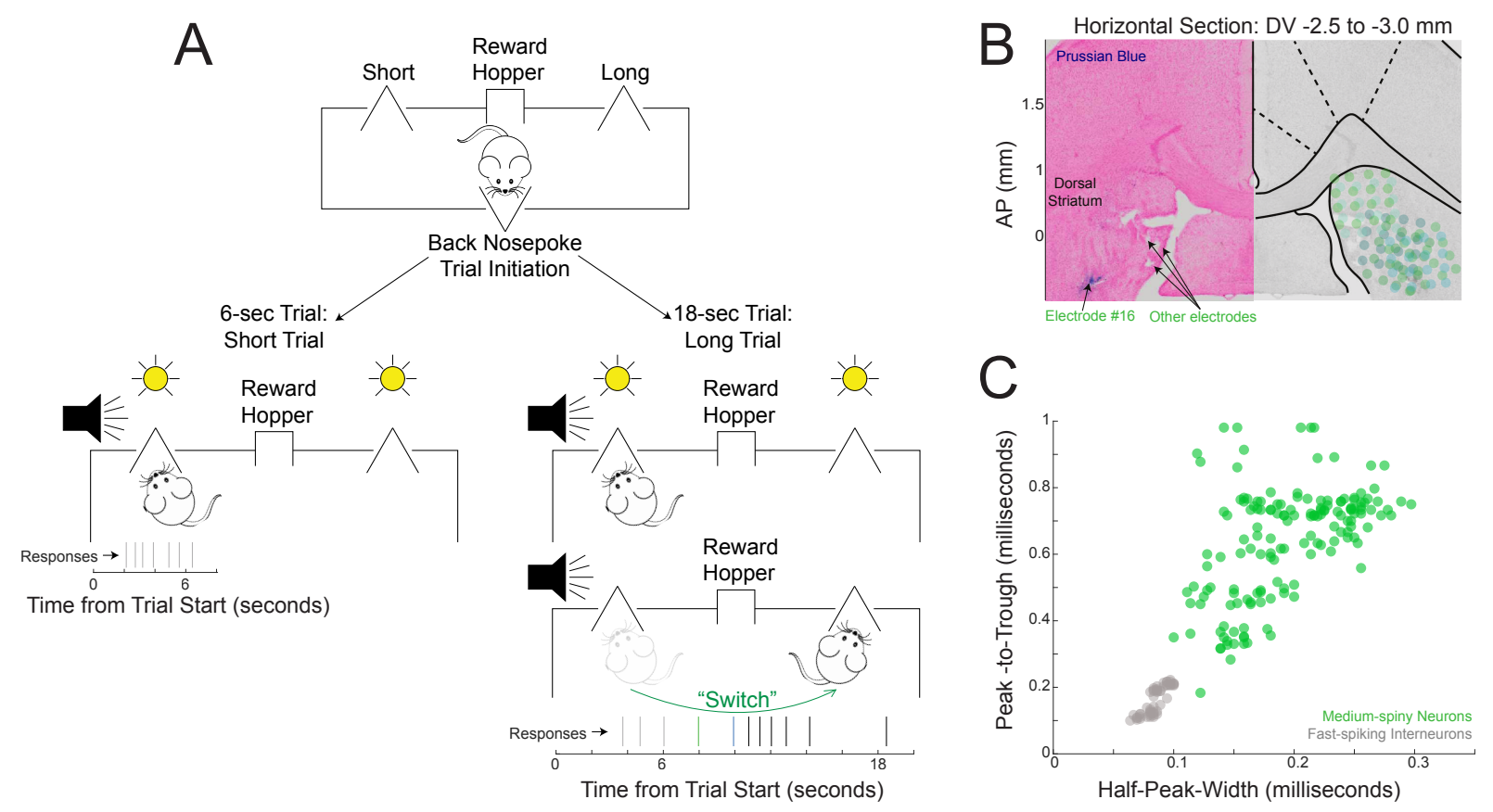

Figure 1: Switch interval timing task and electrode locations. A) We trained animals to

458 perform a switch interval timing task in which mice initiate trials at a rear nosepoke. For $50 \%$ of

459 the trials, mice were rewarded at the short nosepoke for the first nosepoke after six seconds; for

460 the remaining trials (termed "switch trials"), mice were rewarded for the first nosepoke after 18

461 seconds at the long nosepoke. The temporal decision to switch from short to long nosepokes is

462 an explicit time-based decision as in other interval timing tasks. Our analysis was focused on

463 switch trials. B) Electrode locations in the dorsal striatum from one animal (stained with Prussian

464 Blue on the left) and all mice (electrode locations in green on the right). C) We identified

465 medium-spiny neurons (MSNs) based on clustering of waveform peak-to-trough distance and

466 waveform half-peak width. Data from six mice. 

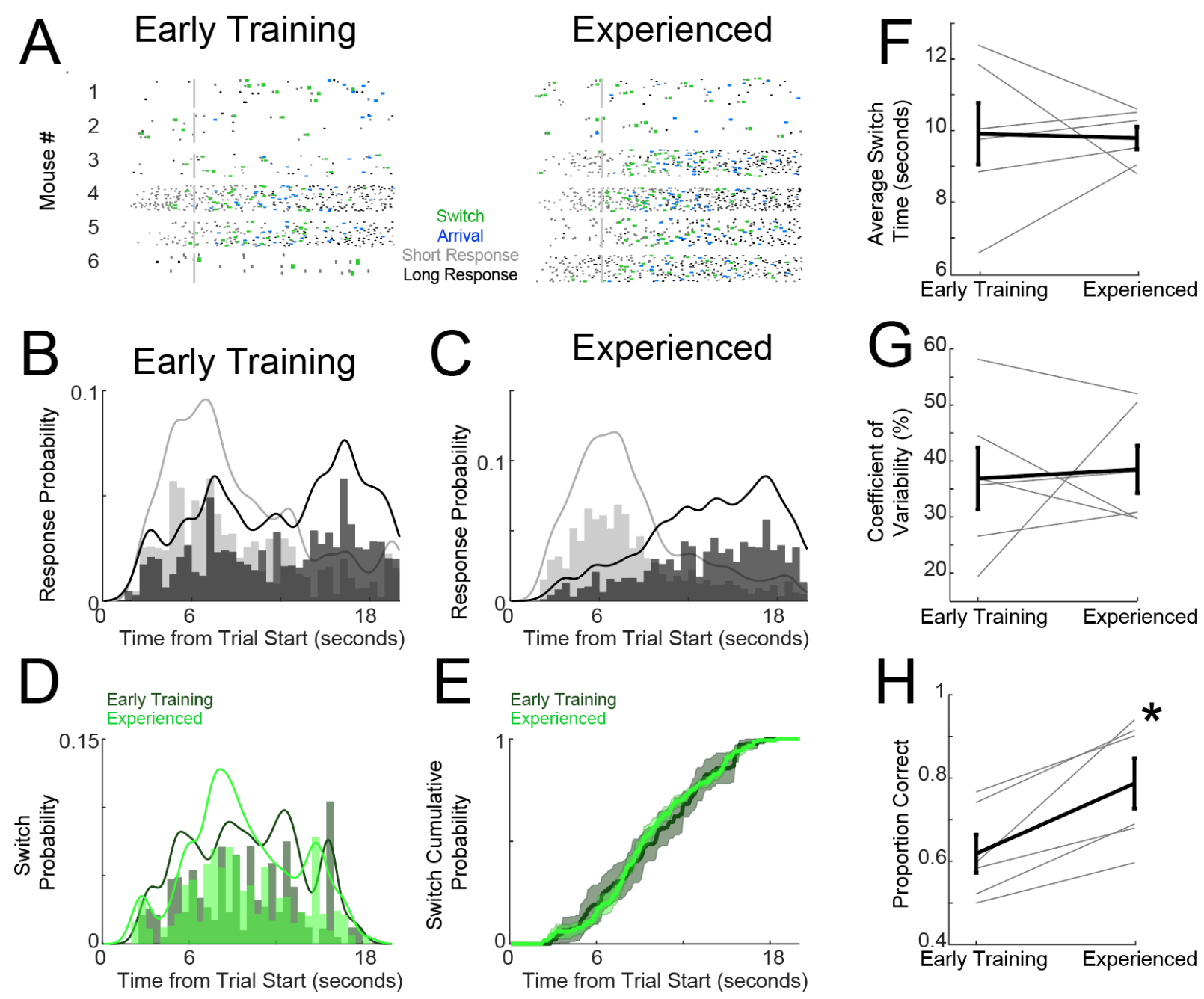

Figure 2: Interval timing performance improves with experience. Rasters of short nosepokes

470 (gray), long nosepokes (black), and switch nosepokes (green) in (A) early training vs.

471 experienced animals. B) Time-response histograms of short (gray) vs. long (black) nosepokes for

472 early training vs. C) experienced animals. D) Distribution or E) cumulative probability of switch

473 responses (when animals departed from the short nosepoke and subsequently responded at the

474 long nosepoke) did not change with experience. F) Mean switch times or G) the coefficient of

475 variability did not change with experience. H) The proportion of correct responses increased with

476 experience. Gray lines in F-H are individual animals; black lines are mean \pm SEM. $*=p<0.05$ via

477 Wilcoxon signed-rank. Data from 445 trials in six animals. 

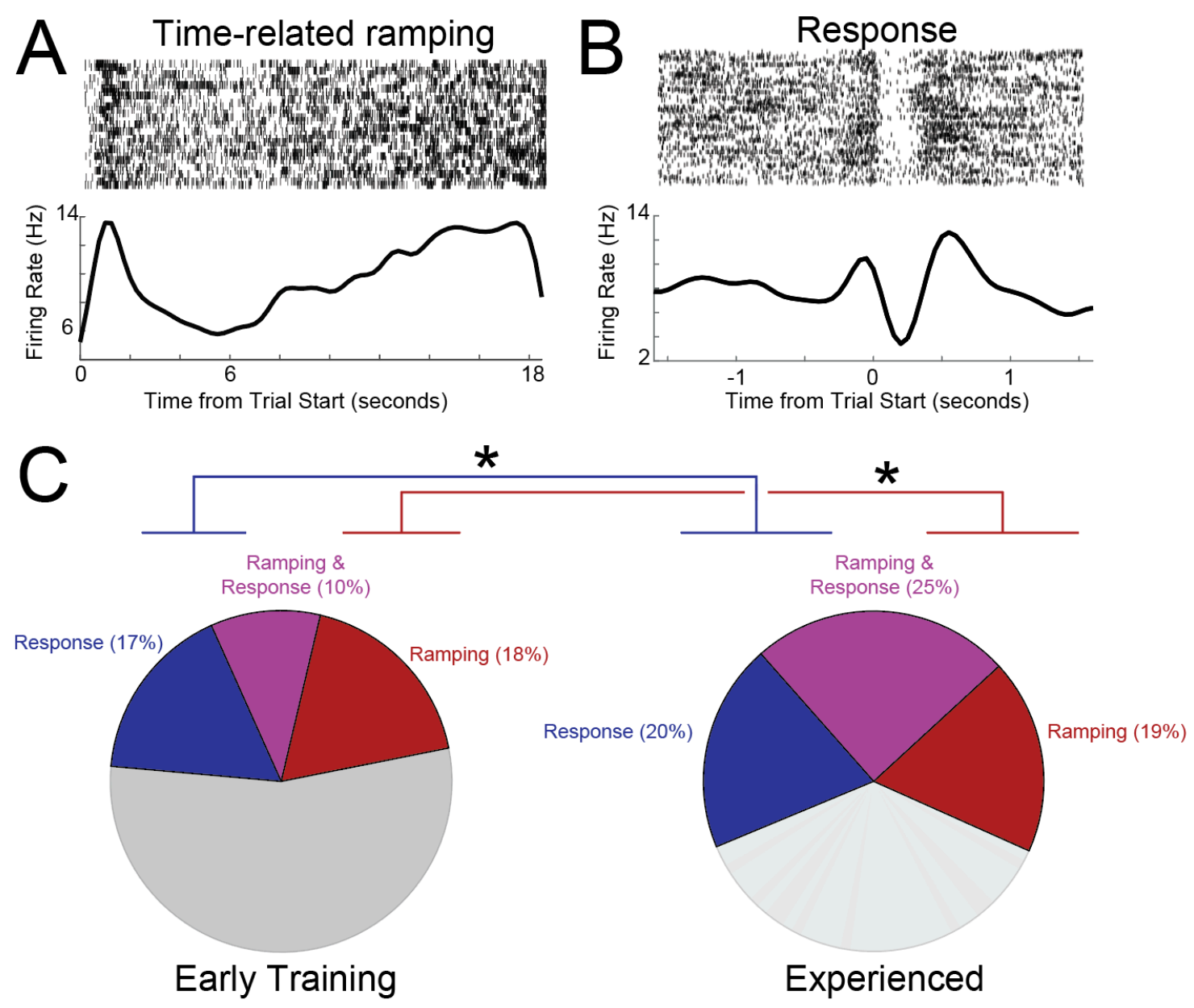

479

480

481

482

483

484

485

486

487

488

489

Figure 3: Response-related and timing-related MSNs increase with experience. A) Example

of an MSN with time-related ramping activity. B) Example of an MSN with response-related activity. C) Pie charts illustrating the frequency of MSNs exhibiting significant response-related activity (blue), time-related ramping activity (red), or both (purple), during interval timing in early training vs. experienced sessions. * by ramping- and response-related activity indicates that they both increased in experienced sessions with $\mathrm{p}<0.05$ as calculated by $\mathrm{X}^{2}$-test. Data from 80 MSNs in six mice during early training sessions and 81 MSNs in the same six mice during experienced sessions. 

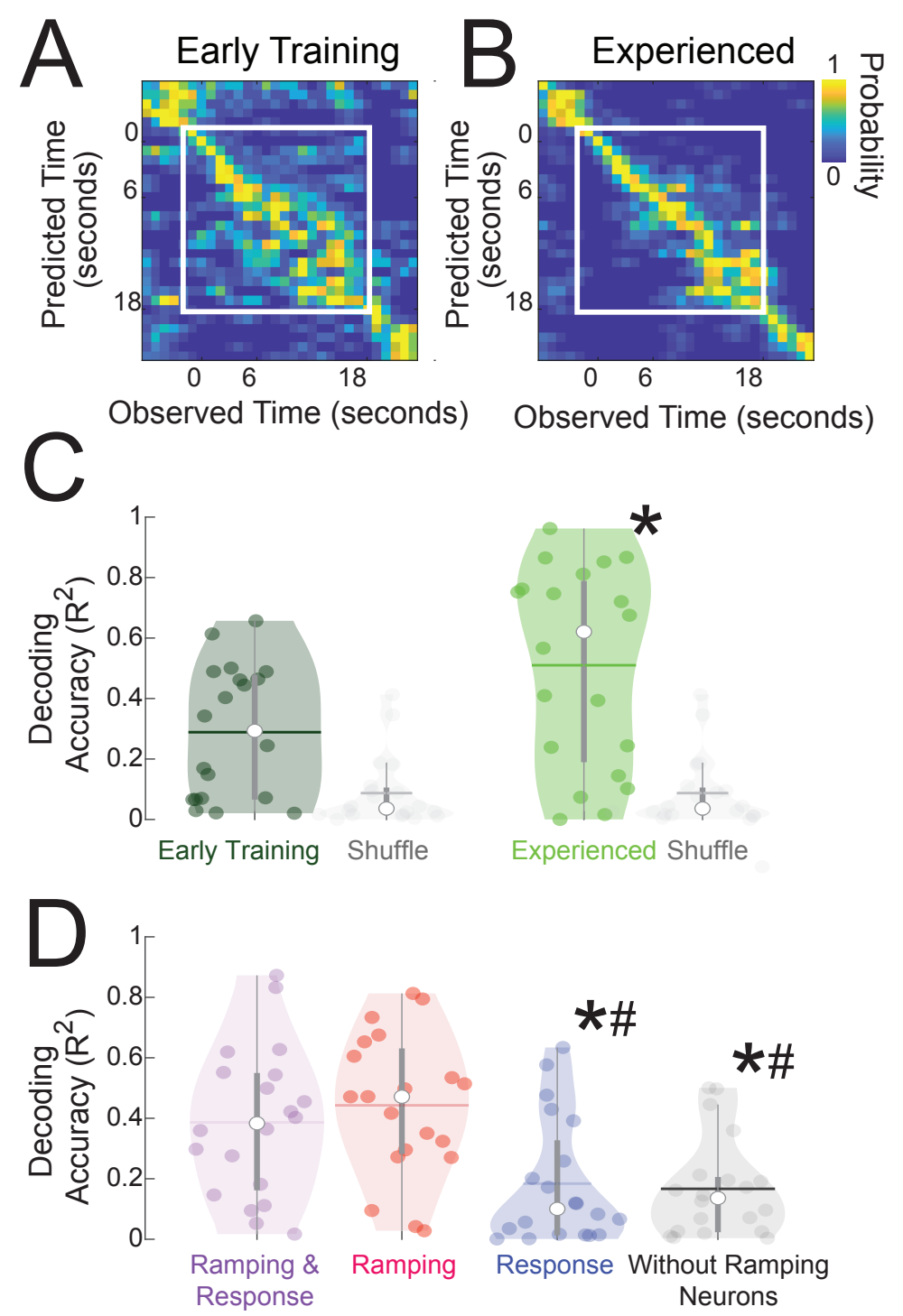

492 Figure 4: MSN ensembles improve temporal decoding with experience. We trained naïve

493 Bayesian classifiers to predict time from firing rate on a trial-by-trial basis. Temporal predictions

494 for A) early training and B) experienced sessions; predicted time is on the y-axis and observed

495 time is on the $\mathrm{x}$-axis, with yellow representing the highest probability. Only the time during the

496 trial (0-18 seconds; white box) is analyzed. C) Temporal decoding improved for early training

497 vs. experienced sessions and was consisently stronger than shuffled data. $*=\mathrm{p}<0.05$ via Wilcoxon 
498 signed-rank. D) Temporal decoding was higher for MSNs with both time-related and response-

499 related modulations than for MSNs with response-related modulations. $*=p<0.05$ via Wilcoxon

500 signed-rank vs. ramping \& response neurons, and $\#=\mathrm{p}<0.05$ via Wilcoxon signed-rank vs.

501 ramping neurons. Critically, MSNs with only response-related modulations were significantly

502 worse for classification than MSNs with ramping or ramping \& response-related modulations;

503 without ramping neurons, temporal decording accuracy decreased. Data from MSN ensembles in 504 six animals during early training and experienced sessions.

505

506

507 

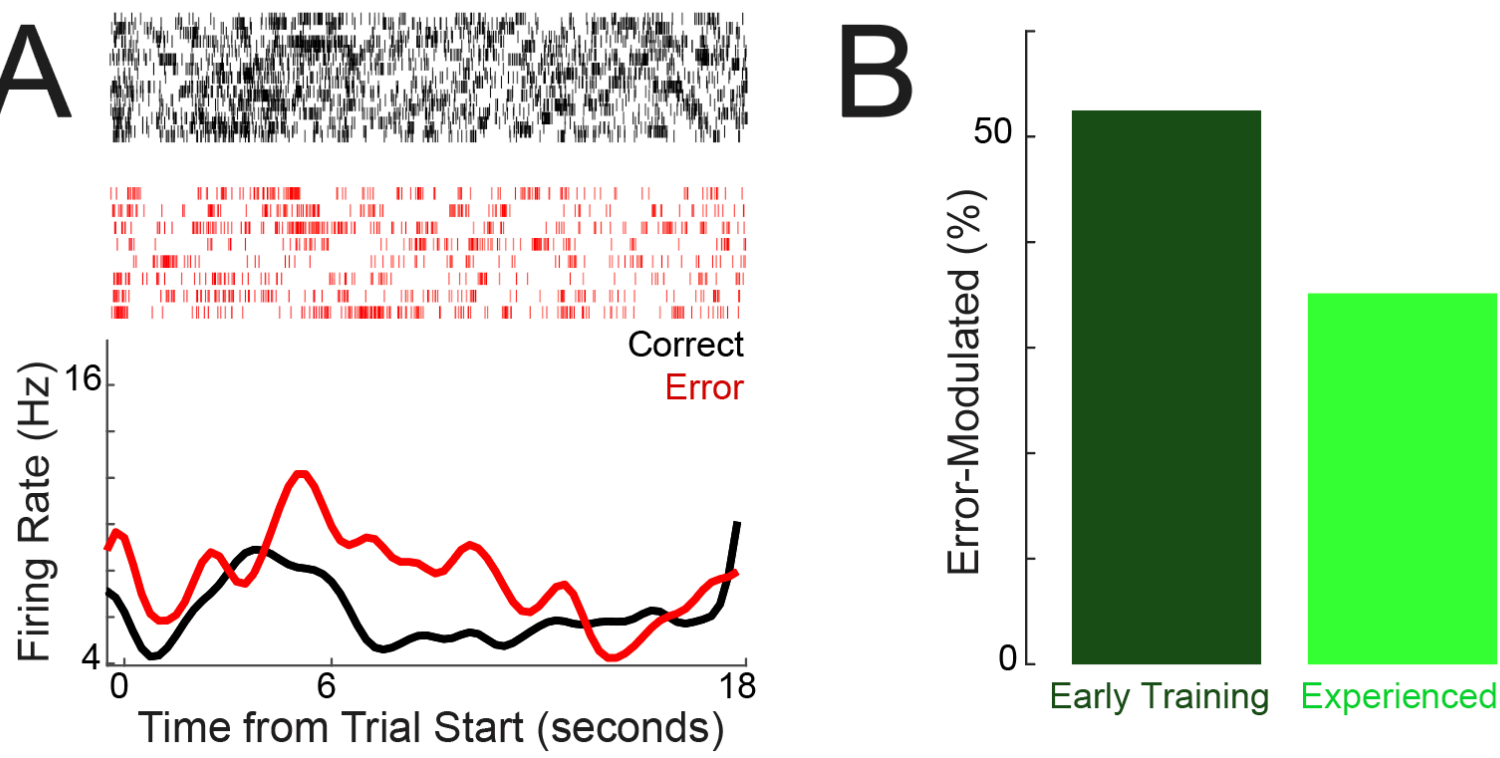

Early Training Experienced

Figure 5: Neuronal activity on error trials. A) An MSN with distinct activity during error-

511 related trials. B) Fraction of error-trial modulated neurons in early training vs. experienced

512 sessions. Data from 98 neurons with $>5$ error trials in six mice during early training and

513 experienced sessions. 